\title{
Quaderni
}

QUADERNI Communication, technologies, pouvoir

\section{Administrer la participation : l'invention d'un métier entre valorisation du militantisme et professionnalisation de la démocratie locale}

\section{Alice Mazeaud}

\section{(2) OpenEdition}

\section{Journals}

Édition électronique

URL : http://journals.openedition.org/quaderni/621

DOI : 10.4000/quaderni.621

ISSN : 2105-2956

Éditeur

Les éditions de la Maison des sciences de l'Homme

\section{Édition imprimée}

Date de publication : 5 octobre 2012

Pagination : 45-58

Référence électronique

Alice Mazeaud, « Administrer la participation : l'invention d'un métier entre valorisation du militantisme et professionnalisation de la démocratie locale », Quaderni [En ligne], 79 | Automne 2012, mis en ligne le 05 octobre 2014, consulté le 19 avril 2019. URL : http://journals.openedition.org/quaderni/621 ; DOI : 10.4000/quaderni.621 


\section{$D$ ossier}

\section{Administrer la}

participation :

l'invention d'un

métier entre

valorisation $\mathrm{du}$ militantisme et professionnalisation de la démocratie locale

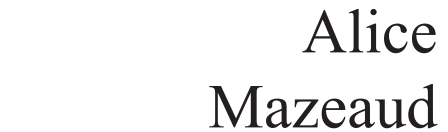

CEJEP Université de La Rochelle
Au cours des quinze dernières années, la participation s'est professionnalisée. Dans les premiers temps, ce sont des militants associatifs et/ou de la politique de ville qui se sont reconvertis en consultants dans le domaine de la démocratie locale et de la participation des habitants ${ }^{1}$. Mais la multiplication de dispositifs participatifs à l'échelle locale, répondant aux obligations législatives (par exemple, les conseils de quartiers dans les communes de plus de 80000 habitants) ou produits de l'expérimentation des collectivités locales, a fait émerger une nouvelle catégorie de « professionnels de la participation »-les agents territoriaux de la participation - qui constitue le signe le plus tangible de l'institutionnalisation d'une offre publique de participation. On ne peut davantage prétendre déterminer le nombre de ces agents qu'espérer quantifier les dispositifs participatifs locaux (plusieurs milliers). Avant cela, il faudrait déjà identifier les contours de ce groupe. Qui sont et que font les agents territoriaux en charge de la participation? En dépit du foisonnement d'études sur la démocratie participative, il semble impossible de répondre de façon satisfaisante tant des acteurs aux profils et aux statuts hétérogènes se retrouvent dans l'appellation « professionnels de la participation », et tant il existe des connexions étroites et des circulations fréquentes entre les « professionnels» du secteur privé et ceux du secteur public.

Même en se limitant à l'étude des agents territoriaux, fonctionnaires ou non, le terme de « professionnel de la participation » contribue à unifier un groupe dont la diversité des statuts et des appartenances demeure manifeste. Qu'ont en commun ceux qui « font » de la démocratie participative à plein temps et ceux qui mettent en place 
des outils de concertation dans le cadre de leur activité ordinaire ? Si l'on a bien vu apparaître des chargés de mission « démocratie participative », « démocratie locale » ou « concertation » formés, pour certains, dans le cadre de formation supérieures spécialisées, ils ne constituent qu'une fraction, la plus visible et sans doute la plus revendicative, des agents territoriaux de la participation. La voie royale (formation spécialisée/concours de la fonction publique territoriale) constitue encore l'exception, et l'apprentissage de terrain, la formation sur le tas et la reconversion de dispositions professionnelles et militantes, demeurent l'ordinaire de l'accès au métier. En ce sens, à l'image d'un groupe de professionnels du gouvernement démocratique dont les frontières sont plus labiles que jamais, rassemblant agents territoriaux, consultants, spécialistes de sciences sociales ou de la communication, la fonction d'agent territorial de la participation est en voie de professionnalisation.

Aussi l'objet de cet article est de montrer comment, malgré l'absence de formations communes ou de fiches de poste standardisées, se constitue un ensemble de savoir-faire et de savoir-être identifié comme spécifique aux agents territoriaux de la participation. Et surtout comment ces agents cherchent à construire sa légitimité dans la valorisation d'une croyance en la démocratie et en la nécessité d'un changement de pratiques au sein des collectivités locales.

Ces processus de construction de pratiques et de savoir-faire propres à la fonction d'agent territorial de la participation et de légitimation/ délégitimation de la fonction seront retracés à partir de deux entrées successives. La première résulte d'un travail d'enquête sur la démocratie participative en Poitou-Charentes ${ }^{2}$ et notamment sur la mise en œuvre du Budget Participatif des Lycées (BPL), conçu comme l'un des plus ambitieux dispositifs participatifs français. Elle analysera la formation d'un ethos militant des agents en charge du BPL à travers les multiples prescriptions ordinaires opérées au quotidien. La seconde entrée empirique analyse la socialisation et la professionnalisation des agents territoriaux de la participation à partir de l'observation d'espaces de rencontres dédiés à ces agents ${ }^{3}$. En effet, on peut interpréter l'organisation de sessions de travail consacrées aux « professionnels de la participation » et à leur relation avec les élus et les associations, comme le signe tangible d'un processus en cours de construction d'une identité et d'un référentiel professionnels. Le croisement des analyses souligne que le métier d'agent territorial de la participation se constitue dans la production d'une figure commune - « le mili-techni $»^{4}$ - construite en opposition nette à la figure du bureaucrate-wéberien et qui entremêle valorisation de savoir-faire spécifiques et de dispositions militantes. Sous-produit de la faible institutionnalisation de la participation à l'échelle locale, la valorisation du militantisme et de la croyance des agents nous éclaire sur l'entreprise de légitimation de la participation au sein des collectivités locales.

\section{L'ethos militant des agents du Budget Participatif des Lycées}

En quelques années, suite à l'élection en 2004 de Ségolène Royal à sa présidence, la région Poitou-Charentes s'est positionnée aux avantpostes du thème de la démocratie participative 
grâce à la multiplication des expérimentations participatives $^{5}$. Le BPL en constitue la figure de proue. C'est sur la base de cette procédure que s'est formée et soudée une petite équipe d'agents, composée de cinq à dix personnes, en charge de la démocratie participative au sein du Conseil régional. En effet, afin de souligner le caractère transversal de la démocratie participative, Ségolène Royal et sa plus proche conseillère ont fait le choix de ne pas créer de service dédié à la démocratie participative. Ainsi, d'un point de vue institutionnel, la chargée de mission « démocratie participative » est rattachée à la direction de l'Education, secteur de déploiement du BPL. Les frontières du groupe des agents en charge du BPL fluctuent suivant les besoins d'animation ou les évènements à organiser. Les évolutions de carrière des uns et des autres peuvent également les conduire à ne plus s'occuper du dispositif. Ainsi, plus que dans les frontières administratives, c'est dans le profil et la socialisation des agents que réside l'unité de l'équipe. Les agents du BPL sont des " généralistes », des cadres de catégorie A, plutôt jeunes (la plupart a environ 30 ans) qui, dans le cadre de leur socialisation institutionnelle ont incorporé un ensemble de représentations et de pratiques spécifiques, que nous qualifions d'ethos militant ${ }^{6}$, pour souligner qu'il est autant la condition que le résultat de leur travail.

\section{Des agents disposés au militantisme}

Malgré des statuts divers - fonctionnaire d'État en détachement, fonctionnaire territorial, chargé de mission - tous ont été recrutés pour leur compétence dans le domaine concerné par leur mission (la restauration collective, la culture, la démocratie participative, la jeunesse...) et pour leurs dispositions personnelles. Des qualités d'animation et d'écoute du public, mais aussi un intérêt pour les enjeux politiques et une disponibilité pour s'engager sont alors recherchées, ce que souligne le directeur des Lycées :

«Là ce sont tous des gens qui partagent les mêmes valeurs, qui sont prêts à s'investir. On n'a jamais demandé la carte, mais on sentait bien les sensibilités. Un truc comme ça, ça peut se faire qu'avec des passionnés qui prennent des risques, qui comptent pas leur temps. C'est des passionnés les gens qui sont là (...). D’ailleurs ça a posé problème, on ne trouvait personne en interne, on ne recrutait que des contractuels $»^{7}$.

L'entrée dans l'administration d'agents prêts à s'engager pour le dispositif est perçue comme une condition de mise en œuvre de la démocratie participative. C'est du reste sur le mode de l'engagement militant que ces agents vivent leur participation au dispositif. Le fonctionnement de ce dernier exige de sortir du cadre administratif, tant du point de vue des horaires que des déplacements pour aller au près des besoins et des contraintes du public ; ce qui requiert une disponibilité maximale et conduit ces agents publics à se définir comme des militants :

« Oui, ça s'appelle du militantisme...Si on y croit pas, ou si on reste dans un cadre purement administratif, le budget participatif des lycées peut pas exister : on est sur une période concentrée de quatre mois, à peu près, cette année c'était notre défi et il a été tenu... mais ça nécessite d'engranger des heures et de s'investir à fond... je dis pas que par ailleurs on s'investit pas mais ce n'est pas du tout le même rythme de travail $»^{8}$. 
De plus, le dispositif est exigeant nerveusement et émotionnellement. Pour gérer les tensions nées de la mise en œuvre du dispositif ou les critiques des participants sur les aberrations du fonctionnement administratif ou la non-réalisation de certains projets, les agents doivent être réactifs et empathiques. Ils doivent faire face à un public contestataire ou le plus souvent atone, passif. Dans ces deux cas, il faut savoir convaincre de l'intérêt et du bien-fondé du dispositif et pour cela " faut être [soi-même] convaincu $»^{9}$. Même si aujourd'hui les résistances et la contestation sont moindres, l'agent doit savoir argumenter sur la légitimité du dispositif, expliquer les rationalités de la procédure pour faire face aux tentatives de subversions du dispositif ou tout simplement motiver un public sceptique. Ainsi, ils doivent avoir suffisamment intégrer les catégories d'entendement du politique pour se substituer aux élus, souvent absents, dans la défense de cette idéologie participative régionale :

«Oui, faut adhérer à un projet politique qui est porté par la présidente et ça c'est indéniable. (...) Il est vrai que lorsque les élus ne sont pas là, il faut porter la politique régionale dans les établissements puisqu'on est censés en représenter les services... $\gg^{10}$.

Ainsi, à rebours du bureaucrate wéberien idéal typique, on voit que le BPL requiert des agents qu'ils s'engagent avec passion et parti-pris pour la mise en œuvre de la « démocratie participative ». C'est aussi la condition de leur promotion et de leur survie professionnelles. En ce sens, cet engagement est un sous-produit de la faible institutionnalisation du dispositif. D'un côté, l'organisation du BPL requiert un investissement maximum des agents et de l'autre, en tant que représentants de l'institution, ils sont amenés à convaincre le public de la grandeur du dispositif. Les pratiques dominantes au sein du service sont donc fortement politisées, tous les agents ayant aussi été recrutés pour leur croyance en la vertu de la participation citoyenne et donc leur adhésion à l'idée centrale du programme de la majorité régionale. Si certains étaient engagés politiquement, facilitant par là même leur entrée dans l'institution ou leur promotion, ces agents n'étaient pas particulièrement connaisseurs en matière de « démocratie participative ». Pour ceux dont la politisation initiale était plus résiduelle, c'est la connaissance d'un des agents en poste qui leur a permis d'obtenir un entretien. Mise à part la chargée de mission « démocratie participative», aucun de ces agents n'était spécialiste de la «démocratie participative ». En d'autres termes, ce ne sont pas leurs connaissances ou leurs compétences en matière de participation, acquises au cours d'une carrière militante ou professionnelle, qui furent à l'origine de leur recrutement mais des dispositions plus générales au militantisme.

\section{L'apprentissage d'un savoir idéologique et pratique de la démocratie participative}

$\mathrm{Au}$ moment de leur entrée en fonction, ces agents bénéficient d'une formation interne sur la « démocratie participative », pour apprendre les justifications politiques de cette alternative politique, découvrir la diversité des expériences et les difficultés liées à leur mise en œuvre, ainsi que la place occupée par le BPL dans le monde des innovations participatives. Ces journées donnent à ces agents profanes en matière de « démocratie 
participative », des clefs de compréhension et de promotion du dispositif qu'ils allaient devoir porter dans les établissements scolaires; ils y apprennent notamment à parler de « crise de la représentation », de « savoir de l'usager », de «délibération collective » etc. De plus, la plupart des agents assiste au colloque annuel sur la « démocratie participative » organisé par le conseil régional ; ils bénéficient donc de la collaboration régulière entre la chargée de mission « démocratie participative » et les spécialistes de sciences sociales engagés sur le sujet.

En parallèle, ils ont fait l'apprentissage du terrain ; en binôme tout d'abord, seul ensuite. Pour les aider, quelques réponses types à des questions/ critiques pièges, ou délicates, ont été élaborées. Par exemple, comment répondre à : «le $B P L$ est illégitime car il concurrence les instances représentatives de l'établissement ", "pourquoi les travaux n'ont pas été réalisés? ", ou encore «le BPL est une stratégie électoraliste » etc.

Mais ce sont surtout les régulations informelles, lors des déjeuners, ou des discussions de couloirs, qui s'avèrent déterminantes, car elles permettent de partager la satisfaction d'une réunion réussie ou de savoir comment faire en cas de contestation; les leaders les plus politisés qui connaissent le mieux l'histoire du BPL, comme la chargée de mission « démocratie participative » ou le chef de service, jouent alors un rôle essentiel. Ils réexpliquent les justifications politiques à apporter, ils partagent leur expérience, ils rassurent, ils remotivent et en même temps ils imprègnent les moins politisés de leur connaissance de l'action régionale. Parfois même au fil des discussions, les slogans régionaux - «le gagnant-gagnant»,
« un euro dépensé = un euro utile », "partager les décisions pour prendre les bonnes »-sont énoncés comme des réponses prêtes à penser lors des réunions participatives. C'est surtout à travers cette socialisation diffuse que se transmet l'essentiel des savoirs et des pratiques, et donc que s'opère de la politisation des agents.

La production d'un ethos militant souligne que l'ensemble des valeurs et des pratiques de ces agents est autant la condition que le résultat de leur travail : ils doivent expliquer, justifier, défendre la politique et le dispositif dont ils ont la charge, ce qui suppose des ajustements et des apprentissages incessants. Bien qu'elle ne se traduise pas toujours par une promotion hiérarchique, la participation au BPL correspond pour ces agents à une carrière ascensionnelle. Les fonctions liées à la gestion et à l'animation du BPL sont caractéristiques des «métiers flous » faits de statuts bricolés, d'une large autonomie dans la définition et l'exercice de la fonction et d'une ambigüité de la relation avec les élus ${ }^{11}$. Ainsi, les agents du BPL sont pour la plupart des chargés de mission, ce qui permet un recrutement sur mesure et des « rémunérations correctes ${ }^{12}$. Rejoindre le BPL, c'est intégrer un service administratif doté d'une forte valeur symbolique - s'occuper des citoyens, servir l'idéal démocratique - distribuant des rôles, des tâches, valorisés et valorisant, qui peuvent faire l'objet de rétributions symboliques et de louanges publiques. C'est jouir d'une visibilité et d'une reconnaissance du public qui a peu d'égal au sein de l'administration régionale ${ }^{13}$, et c'est dans le cas particulier de la région PoitouCharentes participer à une action reconnue comme étant de premier plan. En outre, participer au BPL, c'est intégrer un service administratif 
au fonctionnement plutôt horizontal fondé sur l'autonomie et la responsabilité individuelle et se « rapprocher du politique » grâce à un contact régulier avec les élus, et à la relation entretenue avec la conseillère spéciale de la présidente du Conseil régional ${ }^{14}$. La fonction elle-même, c'est-à-dire les tâches à exécuter, est aussi perçue comme bien plus gratifiante que l'ordinaire de la fonction administrative. Ils décrivent ainsi leur mission d'animation de réunion sur le mode du « plaisir », de « l'excitation », et dessinent une figure archétypale du fonctionnaire - l'agent « administratif » qui « fait de l'instruction de dossiers »-qu'ils érigent en figure repoussoir :

«Mon job c'est porter une démarche qu'est, qu'est validée par l'exécutif régional et qu'est politisée quoi qu'on en dise....plus par exemple que si, que...elle est plus politisée que d'autres services qui ne font que de l'instruction de dossiers, et qui donnent des subventions. Là c'est de l'administratif, nous on n'est pas sur ce champ là. (...) C'est plutôt sympa, moi j'me ferai chier à faire du traitement de dossier administratif à la con. C'est ça qu'est sympa, parce que chaque réunion c'est une nouvelle, y'a pas une réunion qui ressemble à une autre $»^{15}$.

Dans les entretiens, on peut lire la mise en cohérence progressive entre les prescriptions du rôle d'agent public chargé de l'animation du BPL et les représentations des agents concernant le « bon service public ». L'ajustement s'opère entre le système de valeurs et de pratiques de la « démocratie participative »- notamment l'impératif de réactivité - et les dispositions militantes des agents publics qui en ont la charge. En effet, la réactivité entendue dans le sens commun - savoir réagir vite et se montrer disponible, débrouillard - et dans le sens politique (responsiveness) être sensible aux besoins et aux préférences des citoyens - constitue le socle de l'identité professionnelle de ces agents. Si les élus peuvent leur reprocher de « [ne pas avoir] cette culture on exécute les décisions des élus ${ }^{16}$, leur extériorité à l'administration - du point de vue de l'identité et du statut - facilite la prise en charge de la méthode participative érigée en contrepoint des pratiques administratives existantes et l'acceptation de la dimension politique de leur fonction. Elle souligne encore la faible institutionnalisation de cette politique qu'ils contribuent à façonner en définissant les contours de l'identité et des pratiques du service administratif qui en a la charge. Or, pour la plupart, ces agents sont trentenaires, célibataires, contractuels et dépendent de la réussite électorale de l'équipe politique en place, et du succès du BPL «sans [lequel ils] n'existent pas $\gg{ }^{17}$ : tout les pousse à s'engager et à souligner leurs différences, tout autant subies que voulues, avec l'administration plus « classique ». Ainsi, ils définissent leur identité et leurs pratiques en délégitimant celles des services installés réputés refléter le « mauvais service public », enfermés dans leurs routines bureaucratiques et centrés sur leurs propres intérêts que sur ceux du public. Avec le concours des soutiens scientifiques de l'équipe nouvelle, ils incarnent une "modernisation participative »du service public : un service public plus en phase avec les attentes et les citoyens, un service public qui en creux s'oppose à la critique d>une administration opaque, bureaucratique, coupée de la réalité sociale. Par là, ils contribuent à légitimer la redistribution des positions qui s'est opérée à leur profit au sein de l'administration régionale des lycées ${ }^{18}$. 
Les agents territoriaux de la participation : une professionnalisation en chantier

Mettre en perspective ce processus de construction de l'ethos militant des agents BPL avec les échanges observés au sein des espaces de rencontres nationales entre « professionnels de la participation » nous éclaire sur les caractéristiques du métier d'agent territorial de la participation. A priori tout distingue la situation des agents de Poitou-Charentes des agents territoriaux de la participation au sein d'autres collectivités locales. Rares sont les collectivités locales à disposer d'un tel portage politique de la « démocratie participative » et d'une telle proximité vis-à-vis de la recherche académique. Autant d'éléments qui, ajoutés à la dimension collective du travail d'animation du BPL, ont été déterminants dans la production de l'ethos militant des agents du BPL. À l'inverse, lors des rencontres observées, la plupart des agents territoriaux pointent leur isolement et l'absence de portage politique comme difficulté de leur tâche. Pourtant, en dépit des spécificités de la configuration picto-charentaise, l'observation des espaces de rencontre des agents de la participation donne à voir, d'une part, la formation et la consolidation d'une figure commune « le mili-techni » et, d'autre part, la quête partagée de savoir-faire, de ressources pour construire et asseoir la légitimité de la participation au sein des collectivités locales.

\section{"Le mili-techni ", figure commune d'un groupe hétérogène}

La sociologie de ces agents territoriaux telle qu'elle se donne à voir lors de ces rencontres est sans surprise : ce sont majoritairement des femmes, plutôt jeunes, principalement agents de catégorie $\mathrm{A}$. Les voies d'accès à la fonction sont multiples. Si les plus jeunes sont issus de formations spécialisées en gestion des collectivités locales et ont suivi des cours sur la « démocratie participative $»^{19}$, la participation ne faisait pas partie du cursus initial des deux-tiers des agents présents $^{20}$. La plupart d'entre-eux occupe ce métier suite à un processus de (re)conversion dans la participation, après avoir entamé leur carrière au sein d'un autre service d'une collectivité locale (dans les services communication, dans les administrations sectorielles du type environnement) ou à l'extérieur (secteur associatif, journalisme).

Au-delà de ces trajectoires diverses, qu'il s'agirait de préciser à partir d'enquêtes quantitatives plus fines, ces agents ont en commun d'exercer un métier faiblement institutionnalisé tant du point de vue des savoir-faire et des savoir-être requis que de sa position institutionnelle. Du reste, l'hétérogénéité des carrières, des trajectoires, des fonctions et des statuts des agents qui se sont reconnus dans le terme de " professionnels de la participation » constitue un révélateur du bricolage institutionnel auquel ils sont contraints. Parmi les inscrits aux rencontres de ces professionnels organisée dans le Val-de-Marne ${ }^{21}$, les différences sont sensibles tant dans l'appartenance institutionnelle (70\% issus de mairie, $11 \%$ d'agglomération, $16 \%$ de conseil général, et $4 \%$ de conseil régional) que dans la hiérarchie des postes $(64 \%$ de catégorie A, $21 \%$ de catégorie $\mathrm{B}$, et $6 \%$ de catégorie C). Au-delà des grades, ce sont les intitulés des postes qui mettent en évidence la plus ou moins forte division du travail lié à la participation au sein des collectivités et les différences de positions institutionnelles. On 
peut tout d'abord penser à la différence entre ceux qui travaillent, voire gèrent, un service dédié à la participation $(30 \%)$, pour qui la participation constitue le cœur de métier, et ceux qui travaillent au sein de direction sectorielle, pour qui elle constitue une méthode parmi d'autres à mettre en œuvre. Dans les dénominations remplies par les participants pour définir leurs fonctions, on observe ainsi pas moins de cinquante qualificatifs différents : animateur (« développement local», « démocratie locale », « insertion »), chargé de mission (« concertation », « démocratie de proximité », « participation des habitants ») ou directeur (DGA, directeur de la communication). Certaines appellations, à l'image de l'opposition entre concertation et démocratie participative, peuvent traduire des différences profondes dans la conception de la participation et de ses fonctions $^{22}$. Mais cette différence idéologique - le terme participation fonctionnant comme un totem devant lequel tous se retrouvent - n'est pas la seule à être invisibilisée dans les moments de rencontre entre professionnels; les différences de statut le sont aussi. Le rapprochement d'agent de catégorie $\mathrm{C}$ « animateur de concertation », de « directeur de la démocratie locale » catégorie A et de chargé de mission au cabinet reflète combien la catégorie d'agent territorial de la participation dissimule la division du travail pourtant bien réelle entre ceux qui animent les dispositifs obligatoires et ceux qui, à l'image de la chargée de mission démocratie participative de la région Poitou-Charentes, conçoivent les expérimentations de demain.

Alors que tout pousse à distinguer les situations des agents, on peut noter que l'absence de cadre institutionnel et professionnel conduit ces agents à inventer au quotidien les éléments constitutifs de leur métier. Notamment ils se construisent sur la base de postures et de figures communes, celle du médiateur entre élus/administration/citoyens et surtout celle du « mili-technicien », figure hybride dressant le portrait d'un professionnel à la fois engagé dans la conception, la réflexion et la mise en œuvre concrète (celui qui tient le stylo, le micro) :

«Les compétences relèvent à la fois du savoirêtre et du savoir-faire. Les qualités de savoir-être correspondent à la capacité d'écoute, à la dimension relationnelle, au respect et à la passion $d u$ métier, tandis que le savoir-faire consiste en la capacité de mise en réseau, la communication et l'animation. Les besoins portent d'une part sur les outils et les méthodes permettant d'entrer en relation avec les habitants, et d'autre part sur le portage politique $»^{23}$.

L'imprécision et le flou sur les savoir-faire requis sont parés des atours de la plasticité, de l'adaptabilité de l'agent face aux situations les plus diverses. Ils sont aussi l'expression d'une attente de formation, d'échange de bonnes pratiques et de reconnaissance du métier. Ce dont témoigne l'observation de nombreux échanges de pratiques, d'outils et qui a aussi été pointé dans l'évaluation parmi les « éléments qui aideraient les professionnels de la concertation à poursuivre leur action [...] : le besoin de diffusion des expériences, d'échange des pratiques et de partage de méthodes ; une meilleure reconnaissance des métiers de la concertation pour les collectivités locales; le besoin de formations, tant pour l'administration que pour les élus, sur les méthodes de concertation, l'animation et la gestion des 
conflits; une culture de la concertation davantage partagée au sein de la collectivité $»^{24}$.

D'une façon générale, ces Rencontres sont conçues et fonctionnent comme des moments de formation. Pour la plupart des agents, ces moments d'échanges ont constitué des temps forts de leur auto-formation à la participation (ils l'indiquent comme tel dans les questionnaires); ils peuvent d'ailleurs faire valider leur participation au titre de la formation continue (les deux-tiers des participants l'ont demandé). Au-delà de cette validation formelle, les agents déclarent apprécier de découvrir de nouvelles méthodes, et d'ailleurs dans leur organisation même, ces rencontres sont conçues comme une démonstration de méthode et de savoir-faire d'animation (usage du portrait chinois, de la "ronde des envies », de l'enquête géante, des post-it, etc.). Cette quête des « bons outils » est aussi à rapporter au besoin de faire reconnaitre des compétences, des savoir-faire spécifiques aux métiers de la participation vis-àvis des élus et des autres services.

\section{La croyance, contrepoint de la fragilité de la position institutionnelle des agents}

Cet enjeu apparaît de façon saillante dans la valorisation collective de l'engagement militant dans le métier. Le groupe se soude dans la difficulté que chacun expérimente individuellement au quotidien. Pendant les Rencontres, les agents ont applaudi les références à la « galère », soulignant ainsi que les «professionnels de la participation» se construisent avant tout « contre ». Plusieurs ateliers ont ainsi insisté dans leur synthèse pour montrer que ce qui réunit c'est l'impression de lutter, d'user des «forceps en interne », ou encore que "l'important c'est de se connaitre soi-même, se rendre compte que l'on éprouve les mêmes problèmes, les mêmes difficultés ». Pour beaucoup, les épreuves du métier sont le pendant inévitable de la faiblesse de leur position institutionnelle et d'une mission qui s'apparente «à un OVNI dans le paysage politique $»^{25}$. En effet, si la multiplication des dispositifs participatifs locaux a fait évoluer la perception de la démocratie participative au sein des collectivités locales « certains autres services demeurent parfois dubitatifs face aux porteurs de la démocratie participative, considérés comme des 'épileurs de chenille'». Dès lors, beaucoup d'agents se vivent encore comme une " avant-garde » d'agents publics censés convaincre les élus, qui se voient souvent reprochés le manque de soutien des élus, et les collègues, au besoin par la force. Ainsi, la thématique du choc des cultures, du « décalage entre la culture participative et la culture hiérarchisée et technocratique des collectivités $\gg^{26}$ revient de façon constante :

«Moi je crois que dans la pratique, les relations avec les élus, les services, c'est beaucoup plus difficile que ce qui est dit ici. La masse des fonctionnaires, elle est pas engagée, au contraire elle est très insécurisée par ces pratiques. Ici c'est la face émergée de l'iceberg, ceux qui sont très impliqués, les autres le sont beaucoup moins. Tout l'enjeu c'est d'agir au-delà du cercle des convaincus $»^{27}$.

On peut lire dans la valorisation systématique de leur militantisme et de leur souplesse, les attentes d'un groupe qui, à défaut de faire reconnaitre avec succès ses savoir-faire, ses fonctions et surtout ses résultats - ce qui rendrait sa présence 
incontournable - tente de légitimer sa présence par son engagement. Le registre de la croyance est omniprésent : ces agents se définissent comme militants convaincus avant d'être des professionnels. L'un des participants de l'atelier « militant, technicien de la démocratie participative », ira même jusqu'à dire : " militant de la $D P$ [démocratie participative], c'est tout simplement obligatoire, on n'est pas militant de la voirie mais de la DP, oui, forcément $»$. Le fait est que la croyance constitue une forme de rationalisation d'un engagement dans le travail, nécessairement au-delà de ce qui est formellement requis :

«Il apparaît que chacun reste motivé par son travail, bien que des risques d'épuisement aient été identifiés, à savoir la solitude et les rythmes de travail ("les semaines de 35 heures sont très rares; l'animation, l'animation et l'évaluation des réunions prennent un temps fou ») ${ }^{28}$.

En revanche, ce militantisme, intrinsèquement politique, tend à être paradoxalement lui-même dépolitisé : «Il apparaît que la démocratie participative est universelle, elle ne correspond à aucune couleur politique et peut être appliquée dans l'ensemble des collectivités ». En ce sens, à l'image des enseignants ${ }^{29}$, ces agents valorisent la dimension politique (la démocratie) du métier mais réfute sa dimension partisane. Par là, ils marquent aussi leur refus de partager la contrainte électorale et leur souhait de voir leur fonction s'institutionnaliser :

«Oui nous sommes des pionniers en train de construire de nouvelles choses, des gens qui s'impliquent, et oui y'a une dimension politique... et faut être d'accord sur le fonctionnement. Mais on a beaucoup parlé du fait qu'il fallait être militant. Et moi je réfute. Au nom de quoi, il faudrait exiger que les gens soient militants. Par contre, il faut que les agents soient d'accord avec un nouveau mode de relations avec les usagers. Et si les gens sont pas d'accord avec cette relation là, alors effectivement ils ne peuvent pas faire de la démocratie participative $»^{30}$.

La dépolitisation de leur engagement constitue une condition de la professionnalisation et donc de leur survie professionnelle. Le refus de référence systématique au militantisme reflète la crainte de fragiliser encore leur position dans l'administration et d'empêcher toute normalisation de la fonction, ce militantisme semblant difficile à concilier avec le statut de fonctionnaire et le devoir de réserve.

S'ils se reconnaissent dans les images de « l'équilibriste » et du " capitaine de galère ", chaudement applaudies pendant les rencontres des professionnels, c'est aussi en raison de la diversité des missions attribuées à la « démocratie participative »; à cet égard, à plusieurs reprises le triptyque des objectifs démocratiques, sociaux et managériaux de la participation ${ }^{31} \mathrm{a}$ été repris par les agents comme base de définition de leurs missions. À la fois technologie de mobilisation politique et instrument d'action publique, la « démocratie participative» se situe au carrefour de la production des politiques publiques (mieux décider) et du travail de relégitimation de la politique (crise de la représentation). Les agents en charge de la participation se définissent comme des médiateurs, des facilitateurs, situés entre les citoyens et la collectivité, afin de « créer du lien social et rendre le citoyen acteur $\rangle^{32}$. Les finalités 
qui sont, selon eux, celles de la participation leur permettent de donner du sens à leur métier, et de justifier, d'abord à leurs propres yeux, leur engagement. Les termes de facilitateur, de médiateur reviennent de façon constante, y compris dans les groupes plus réduits; les réprimandes adressées à un agent pris en flagrant délit de reformulation de la parole d'autrui, attitude jugée « contraire à l'attitude à avoir vis-à-vis des citoyens », témoignent de l'importance de ce qui constitue à la fois un savoir-faire et un savoir-être partagé.

Plus largement, ces agents partagent la difficulté à mobiliser les citoyens, comme en témoignent les demandes récurrentes sur les bonnes pratiques de mobilisation ou le succès des ateliers consacrés à «faire participer les publics éloignés ». En effet, seul le nombre de participants constitue une preuve difficilement contestable de la valeur de l'offre participative et du savoir-faire de l'agent. En raison de cette fonction d'interface entre la collectivité et le citoyen, l'agent de la participation se situe à l'entrecroisement du travail politique de représentation et du travail administratif de production de l'action publique et donc « dans une position nécessairement transversale par rapport aux élus et autres services $\rangle^{33}$. Ils perçoivent ainsi la difficulté « de mettre en place des démarches qui bousculent le partage du pouvoir et les conceptions de la légitimité d'action et de parole de chacun » comme un élément consubstantiel au métier. In fine, dans leur discours, les agents dessinent les contours d'un nouveau rapport au service public, un « service public au service du public » auquel il s'agit d'intéresser les autres : «Si y on est, si on y reste c'est qu'on y croit. Ce qui fonde notre travail, c'est notre relation au service public. Mais on a une définition différente du service public, qui n'est ni celle de la fonction publique et des autres collègues, ni celle des élus $»^{34}$.

\section{Conclusion}

Du point de vue des pratiques comme du sens donné à la « participation », envisager les agents territoriaux de la participation comme un groupe homogène est discutable. En revanche tous construisent leur identité professionnelle sur la base d'une valorisation de leur croyance en la démocratie et d'une dévalorisation des pratiques anciennes du service public. Ils contribuent ainsi à construire et à légitimer une définition nouvelle de l'identité et des pratiques du service public par opposition à une administration appelée à changer de culture. La figure du bureaucrate wébérien autrefois garant de l'intérêt général est aujourd'hui érigée en figure repoussoir au profit d'une figure plus politisée, celle du « mili-techni » médiateur des attentes des citoyens. On pourrait voir dans les savoir-faire et les savoir-être valorisés des caractéristiques propres au «politechnicien », celui réputé posséder l'art de s'occuper des citoyens $^{35}$, mais ce serait oublier que la critique de l'administration est aussi portée par le New Public Management. Ainsi il est remarquable que tout en s'opposant d'un point de vue idéologique, la démocratie participative et le New Public Management sont comparables du point du vue du travail et des fonctions des agents publics, et des qualités personnelles requises (enthousiasme, réactivité, sympathie, initiative $)^{36}$. Comme le note un élu, «c'est [parce que c'est] difficile, [qu'il s'] appuie sur le militantisme des fonctionnaires $»^{37}$. En ce sens, le militantisme des agents de la participation est sans doute davantage le produit de la faible 
institutionnalisation de leur fonction que le signe de valeurs morales particulières; le service public a toujours eu ses « avant-gardes » qui, entrant dans l'administration contribuent à en redéfinir l'identité, les valeurs et les valeurs et les pratiques dans un processus d'ajustement permanent à leurs propres ressources ${ }^{38}$. La valorisation de la croyance en les vertus de la participation et de la démocratie, comme la nécessité d'innover en permanence ${ }^{39}$, forment le socle de l'entreprise de légitimation de la démocratie participative, et de la redéfinition des rapports élus/agents/citoyens. À défaut de pouvoir produire les preuves de l'efficacité de la démocratie participative, ils administrent la preuve de leur engagement et de leur croyance dans ses vertus; au risque d'éprouver des difficultés à normaliser leur statut. Ainsi, l'apparition d'une fonction publique territoriale de la participation souligne qu'indépendamment de ses effets incertains sur la démocratie, l'institutionnalisation d'une offre publique de participation a déjà des effets notables sur l'organisation et la division du travail politico-administratif au sein des collectivités locales.

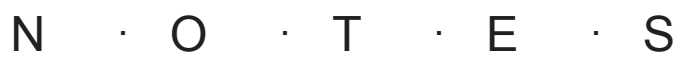

1. M. Nonjon, Quand la démocratie se professionnalise : enquête sur les experts de la participation, Thèse de doctorat en science politique, Université Lille 2, 2006.

2. A. Mazeaud, La fabrique de l'alternance. La démocratie participative dans la recomposition $d u$ territoire régional (Poitou-Charentes 2004-2010), Thèse de doctorat en science politique, Université de La Rochelle, 2010.

3. Ces observations empiriques ont été essentiellement menées lors des Rencontres de la Démocratie Locale en 2010 (ADELS), et lors des Rencontres des professionnels de la démocratie participative destinées aux agents territoriaux organisées par le Conseil général du Val-de-Marne en novembre 2011. Cette dernière observation a été réalisée avec Magali Nonjon, elle s'inscrit dans le cadre d'une enquête commune sur les professionnels de la participation, voir A. Mazeaud, M. Nonjon, « Des fonctionnaires aux prestataires : l'apport de l'observation ethnographique à une cartographie des professionnels de la participation », communication lors du congrès de la Société Québécoise de Science Politique, 2012.

4. Terme élaboré et repris lors des Rencontres de professionnels de la démocratie participative.

5. Y. Sintomer, J. Talpin (dir.), La démocratie participative au-delà de la proximité. Le Poitou-Charentes et l'échelle régionale, Rennes, PUR.

6. Suivant les travaux d'Alexis Spire, la notion wébérienne d'ethos permet de penser des règles incorporées dans le cadre d'une socialisation institutionnelle, et non de dispositions durables et transposables dans d'autres univers. Cf. A. Spire, « Histoire et ethnographie d'un sens pratique. Le travail bureaucratique des agents de contrôle de l'immigration », in P. Fournier 
et al. (dir), Observer le travail, Paris, La Découverte, 2008, pp. 61-76.

7. Entretien, directeur Éducation.

8. Entretien, chef de service BPL.

9. Entretien, chargée de mission Nutrition-Restauration.

10. Entretien, chef de service BPL.

11. G. Jeannot, « Les métiers flous du développement rural », in Sociologie du travail, vol. 47, n¹, 2005, pp. 17-35.

12. Entretien, directeur Éducation.

13. Rappelons que compte tenu des compétences de la région, les agents publics régionaux sont en général peu en interaction avec le grand public.

14. On peut d'ailleurs penser qu'ils partageront la contrainte électorale.

15. Entretien chargée de mission Nutrition-restauration.

16. Entretien vice-présidente Démocratie participative.

17. Entretien, chargée de mission Démocratie participative.

18. A. Mazeaud, «La modernisation participative vue d'en bas : entre militantisme et malaise identitaire », in Pyramides, $\mathrm{n}^{\circ} 18,2009$, pp. 269-290.

19. L'apparition d'un Master spécialisé en ingénierie de la participation témoigne davantage encore de la professionnalisation croissante de la démocratie participative.

20. Professionnels de la participation. Qui sommes nous? Enquête auprès des participants aux rencontres de professionnels de la démocratie participative du Val de Marne.

21. Tout laisse à penser que le public était relativement représentatif de l'ensemble des agents territoriaux de la participation.

22. Ce qui fait l'objet de vifs échanges entre agents lors de ces rencontres.

23. Actes des Rencontres des professionnels de la démocratie participative.

24. Idem.
25. Idem.

26. Idem.

27. Notes d'observation, Rencontres de la démocratie locale.

28. Actes des Rencontres des professionnels de la démocratie participative, op. cit.

29. J. Lagroye, « Les processus de politisation » in J. Lagroye (dir) La politisation, Paris, Belin, 2003, pp. 359-372.

30. Notes d'observation, Rencontres de la démocratie locale.

31. M.-H. Bacqué, H. Rey, Y. Sintomer, « La démocratie participative, un nouveau paradigme de l'action publique ? » in M.-H. Bacqué, H. Rey, Y. Sintomer. (Dir.), Gestion de proximité et démocratie participative : une perspective comparative, Paris, La Découverte, pp. 9-46.

32. Actes des Rencontres des professionnels de la démocratie participative, op. cit.

33. Notes d'observation. Rencontres de la démocratie locale.

34. Idem.

35. L. Mermet, « Débattre sans savoir pourquoi : la polychrésie du débat public appelle le pluralisme théorique de la part des chercheurs ", in Blatrix C. et al. (dir.), Le débat public : une expérience française de démocratie participative, Paris, La Découverte, 2007, pp. 369-380.

36. E. Vigoda, « From Responsiveness To Collaboration: Governance, Citizens and The Next Generation of Public Administration ", in Public Administration Review, vol. 62, n 5, 2002, p. 527-540 ; P. Du Gay, «Without Affection or Enthusiasm'? Problems of Involvement and Attachment in Responsive' Public Management », in Organization, vol. 15, n³, 2008, pp. 335-353.

37. Notes d'observation. Rencontres de la démocratie locale. 
38. B. Gaït, « Histoire d'une renaissance, l'histoire du service public », in Politix, vol. 2, n 6, 1989, 61-67.

39. G. Gourgues, «Les fonctionnaires participatifs : les routines d'une innovation institutionnelle sans fin(s) », in Socio-logos, $\mathrm{n}^{\circ}$ 7, 2012 [En ligne].

\section{$R \cdot E ́ \cdot S \cdot U \cdot M \cdot E ́$}

L'institutionnalisation d'une offre publique de participation s'est traduite par l'apparition d'une nouvelle catégorie d'agents territoriaux ayant spécifiquement pour mission d'administrer la participation (concevoir, organiser, animer les procédures participatives). L'analyse croisée du cas des agents en charge du Budget participatif des lycées de Poitou-Charentes et de rencontres professionnelles des agents territoriaux de la participation, souligne que ces agents, dont la fonction et la position au sein de l'administration sont largement indéterminées, inventent leur métier en érigeant la figure idéal-typique du fonctionnaire wéberien en figure repoussoir. Par leur engagement, ils contribuent ainsi à redéfinir les normes et les pratiques légitimes au sein des administrations territoriales.

\begin{abstract}
The institutionalisation of a public offer of participation has brought about the emergence of a new category of territorial public servants, specifically in charge of administrate the participation (design, organisation, animation of the participatory procedures). A crossed analysis of the case of the public servants in charge of the High Schools Participatory Budget in PoitouCharentes and of the professionals meetings of territorial public servants of participation, underlines that the mission and the position inside the administration of these public servants are quite undeterminated. They invent their profession in opposition to ideal-type of the weberian bureaucracy and by their activism they contribute to redefine the legitimate normes and practices in the territorial administration.
\end{abstract}

